\title{
LAZER, EDUCAÇÃO E MEIO AMBIENTE: UMA AVENTURA EM CONSTRUÇÃO
}

\author{
Humberto Luís de Deus InÁcio*
}

\section{RESUMO}

Este texto busca demonstrar algumas contribuições da temática Lazer e Educação, com atenção à relação entre lazer e meio ambiente, principalmente através das Práticas Corporais na Natureza, apresentando as produções de pesquisadores divulgadas no Congresso Brasileiro de Ciências do Esporte e no Encontro Nacional de Recreação e Lazer. $\mathrm{O}$ artigo também apresenta as contribuições de teses, dissertações, periódicos e livros da área da Educação Física ou próximos dela. Por fim, lista grupos de pesquisa que vêm desenvolvendo pesquisas e projetos relacionando lazer, educação e meio ambiente.

PALAVRAS-CHAVE: educação física - lazer - educação - meio ambiente - práticas de aventura na natureza

\section{INTRODUÇÃO}

objetivo deste texto é apresentar contribuições relativas ao tema Lazer e Educação, focando a relação entre lazer e meio ambiente, traduzida de muitas formas (educação ambiental, políticas públicas, modelos de desenvolvimento, formação profissional e outras), mas principalmente através das Práticas Corporais na Natureza - PA's. ${ }^{1}$ Algumas práticas foram socializadas nos dois mais tradicionais eventos científicos dos quais participam pesquisadores da Educação Física e áreas afins: o Congresso Brasileiro de Ciências do Esporte (CONBRA$\mathrm{CE}$ ) e o Encontro Nacional de Recreação e Lazer (ENAREL).

Também são apresentados, neste trabalho, teses, dissertações, periódicos e livros da área da Educação Física ou próximos dela, além de listar grupos de pesquisa que vêm desenvolvendo pesquisas e projetos, conectando lazer, educação e meio ambiente.

* Professor do Departamento de Educação Física da Universidade Federal do Paraná e doutorando do Programa de Pós-Graduação em Sociologia Política da Universidade Federal de Santa Catarina. 
Este tema foi destacado no estudo de Gisele Schwartz e Jossett de Gáspari (2003), sobre as tendências do Grupo de Trabalho Temático (GTT) "Recreação e Lazer" do Congresso Brasileiro de Ciências do Esporte (CBCE) de 1997 a 2001, apresentado no CONBRACE de 2003: $\mathrm{Na}$ categoria aventura e natureza foram inseridos dezenove trabalhos (19,79\%), que abordavam desde noções de consciência preservacionista a esportes de aventura e relação homem-natureza. Para as autoras, isto reflete um crescimento da oferta de atividades de aventura no lazer e incentivam uma nova relação entre ser humano e natureza.

Para alcançar nosso objetivo, apresentamos o esboço para um "estado da arte" dos trabalhos que abrangem a temática da relação ser humano-meio ambiente nos anais desses eventos a partir de 1999, pois entendemos que daí em diante, de fato, o tema ganha destaque nos debates da Educação Física/Ciências do Esporte. ${ }^{2}$

De forma geral, os textos encontrados apontam para uma "educação pelo lazer", mas também há trabalhos que apontam para um processo de "educação para o lazer". Aliás, concordamos com Reinaldo Pacheco (1992, p. 251), quando nos diz que, assim como outros fenômenos sociais, o lazer é "um espaço de criação e (re)criação de identidades individuais e coletivas". Assim sendo, "com um diálogo apropriado, com estratégias pedagógicas coerentes, com a proposição de uma reflexão contínua e ordenada, acreditamos que as práticas de/no lazer possam caminhar na direção oposta a da reprodução" (INÁCIO et al., 2005, p. 85).

Após a análise dos trabalhos encontrados foi possível agrupá-los em subtemáticas, e algumas delas, via de regra, aparecem juntas num mesmo trabalho: práticas corporais na natureza (a atividade em si, representações, relação atividade-natureza e estilos de vida) - PA's; educação ambiental - EA; conceito de meio ambiente - CMA; impactos ambientais - IA; modelos de desenvolvimento - MD; formação profissional - FP; políticas públicas - PP; e mídia (o papel da..) - MID.

\section{UM ESBOÇO DO ESTADO DA ARTE NOS CONBRACE'S}

A seguir, apresentamos dados e análises dos anais dos CONBRACE's de 1999 a 2005. Os quadros apresentam a quantidade de trabalhos encontrados e a principal subtemática com a qual se relacionam. Isso não significa que outras subtemáticas não estejam presentes: 
Conferências, mesas-redondas, seminários

\begin{tabular}{|c|c|c|c|}
\hline & PA's & FP & MID \\
\hline 1999 & 1 & 1 & 1 \\
\hline 2001 & - & - & - \\
\hline 2003 & 1 & - & - \\
\hline 2005 & - & - & - \\
\hline
\end{tabular}

\section{Grupos de trabalhos temáticos}

\begin{tabular}{|c|c|c|c|c|c|c|}
\hline & PA's & CMA & MD & PP & EA & FP \\
\hline 1999 & 3 & 3 & 2 & - & - & - \\
\hline 2001 & 6 & 3 & 2 & 1 & - & - \\
\hline 2003 & 2 & - & 1 & 1 & - & 1 \\
\hline 2005 & 5 & - & - & 1 & 3 & - \\
\hline
\end{tabular}

Pôsteres

\begin{tabular}{|c|c|c|c|c|c|c|}
\hline & PA's & CMA & EA & FP & MID & PP \\
\hline 1999 & 3 & 1 & 2 & - & - & - \\
\hline 2001 & 1 & - & 1 & 1 & - & - \\
\hline 2003 & 1 & - & 1 & - & - & 1 \\
\hline 2005 & 4 & - & - & - & 1 & - \\
\hline
\end{tabular}

\section{COMENTÁRIOS GERAIS}

Há uma idéia geral de lazer: o lazer se reveste com características consumistas, mas possui caráter revolucionário, que pode ser potencializado por meio das práticas em ambientes diferenciados.

O meio ambiente é visto como a natureza externa: flora, fauna, água, ar, mas em alguns textos há entendimento diferenciado sobre este conceito, apontando para a natureza inorgânica do humano (MARX, 1989). 
Há uma série de debates e contradições teóricas e conceituais; por exemplo, no uso das terminologias "tempo livre" e "tempo disponível".

Há uma indicação predominante de que a cooperação deve ser objetivo das práticas corporais junto à natureza, em detrimento da competição.

A dicotomia ser humano-natureza e a necessidade de sua superação estão bem marcadas.

Em vários trabalhos aparecem conceitos de lazer críticos, mas relacionados a relatos de projetos com tendências utilitaristas.

Há críticas ao modelo hegemônico de desenvolvimento.

Há críticas aos esportes de aventura por seu impacto ambiental.

Há diversos trabalhos/estudos de autores da Educação Física, mas se afastam de certa forma da temática do movimento humano, se aproximando de questões ligadas ao meio ambiente, modelos de desenvolvimento, impactos ambientais, relação ser humano/natureza. Em sua maioria, são estudos de pós-graduação em outras áreas.

Se observa que PA's é a subtemática mais presente em mesas e pôsteres. Em sua maioria, discute-se a relação ser humano-natureza através das práticas corporais de aventura; e é no GTT Recreação e Lazer que se concentra a grande maioria dos trabalhos, exatamente porque, assim entendemos, tais práticas estão muito mais disseminadas no âmbito do lazer do que em outros.

À margem de textos sobre o Lazer, encontramos dois outros, apresentados no CONBRACE/2003, os quais nos chamaram a atenção: o primeiro, apresentado no GTT Escola, é "A influência do Rio Paraguai na cultura de movimento dos munícipes de Cáceres/MT - uma experiência escolar", de Cássia Hack. O outro texto foi apresentado no GTT Memória, Cultura e Corpo por Carmen L. Soares, denominado "Natureza, vida ao ar livre e educação física no início do século XX: fragmentos da obra de Georges Hébert". Parece-nos que os textos apontam outra forma de olhar para o papel educativo no/pelo lazer, mesmo que não tenha sido esse o objetivo de suas autoras.

\section{OS ENAREL'S}

A seguir, dados e análises dos anais dos ENAREL's de 1999 a $2005 .^{3}$ 
Conferências, mesas-redondas, seminários

\begin{tabular}{|c|c|c|c|c|c|c|c|c|}
\hline & PA's & EA & CMA & IA & MD & FP & PP & MID \\
\hline 1999 & 7 & 4 & 8 & 2 & 4 & 2 & 2 & 1 \\
\hline 2000 & - & - & - & - & - & - & - & - \\
\hline 2001 & - & - & - & - & - & - & - & - \\
\hline 2002 & 1 & - & - & - & 4 & - & 1 & - \\
\hline 2003 & - & 1 & - & - & 1 & - & 1 & 1 \\
\hline 2004 & 1 & 1 & - & - & - & - & - & - \\
\hline 2005 & 1 & - & - & - & - & - & - & - \\
\hline
\end{tabular}

Trabalhos de comunicação oral (Mesas Temáticas)

\begin{tabular}{|c|c|c|c|c|c|c|c|c|}
\hline & PA's & EA & CMA & IA & MD & FP & PP & MID \\
\hline 1999 & 6 & 7 & 1 & 1 & - & 4 & - & - \\
\hline 2000 & 4 & 2 & - & 1 & 3 & 1 & - & 2 \\
\hline 2001 & 1 & 1 & - & - & - & - & - & - \\
\hline 2002 & 7 & 5 & - & 3 & 5 & - & 2 & 1 \\
\hline 2003 & 1 & - & - & - & - & - & - & - \\
\hline 2004 & 5 & 5 & - & 1 & - & 2 & - & - \\
\hline 2005 & 9 & 3 & - & - & - & 1 & - & 1 \\
\hline
\end{tabular}

Pôsteres

\begin{tabular}{|c|c|c|c|c|c|c|c|c|}
\hline & PA's & EA & CMA & IA & MD & FP & PP & MID \\
\hline 1999 & 13 & 7 & 1 & 1 & - & 4 & - & - \\
\hline 2000 & 1 & 1 & - & - & - & - & - & - \\
\hline 2001 & 6 & 1 & 1 & - & - & 3 & - & - \\
\hline 2002 & 11 & 16 & 1 & 7 & 3 & 8 & 3 & 1 \\
\hline 2003 & 10 & 1 & - & - & - & - & - & - \\
\hline 2004 & 11 & - & - & 1 & - & - & 1 & - \\
\hline 2005 & 3 & 2 & 1 & 2 & 1 & - & - & 1 \\
\hline
\end{tabular}




\section{COMENTÁRIOS GERAIS}

Há a idéia geral de lazer como espaço de vivências lúdicas com características antagônicas: consumista ou revolucionário, ou dialeticamente com as duas características.

O meio ambiente é visto como a natureza externa: flora, fauna, água, mas em alguns textos se amplia este conceito, resgatando, inclusive, o conceito de natureza humana. Destacamos que no Enarel 2005, esta ampliação do conceito de meio ambiente está mais forte, diminuindo, pelo menos teoricamente, a dicotomia ser humano-natureza.

Há uma série de contradições teóricas e/ou conceituais:

- cooperação x competição, nas práticas corporais junto à natureza;

- rendimento necessário x rendimento obrigatório;

- separação ser humano e natureza;

- conceitos de lazer críticos x projetos utilitaristas no mesmo relato.

Aparece o Sistemismo aplicado às questões ambientais.

Há fortes críticas ao modelo hegemônico de desenvolvimento. Há críticas aos esportes de aventura por seu impacto ambiental.

Também há utilização de referenciais teóricos descontextualizados.

Há diversos trabalhos de estudiosos da Educação Física que se afastam da temática do movimento humano, se aproximando mais de questões ligadas ao meio ambiente, modelos de desenvolvimento, impactos ambientais, relação ser humano/natureza.

No ENAREL 2002, realizado em Natal/RN, a BACOR não apresentou trabalhos, apesar de o grupo estar localizado na mesma cidade.

Pelos quadros, observa-se a temática destacada em "conferências, mesas-redondas, seminários" no ano de 1999. Isto se deve ao fato de o ENAREL daquele ano ter sido realizado em Foz do Iguaçu/PR, território onde a questão ambiental e do lazer fazem parte do cotidiano por sua geografia e economia; o tema central foi "Lazer, meio ambiente e participação humana". A programação principal do evento seguiu essa diretriz.

Observa-se que a subtemática PA's é a mais presente; no entanto, a educação ambiental também se destaca em textos que são da área do Turismo e não da Educação Física, o que aponta uma tendência: os cursos de formação em Turismo estão mais próximos do debate ambiental, principalmente em textos referentes ao ecoturismo e ao turismo de aventura. 
O encontro mais recente (2005) apresentou vários textos que trazem à tona o debate sobre a "ética" na relação lazer-educação-meio ambiente, com destaque para algumas pesquisas realizadas no LEL. Além disso, aconteceu uma mesa de debates específica sobre a relação lazer e meio ambiente.

\section{TESES E DISSERTAÇÕES}

As teses e dissertações a seguir dão mostra de como a produção científica, no âmbito do pelo Lazer, vem incorporando a questão ambiental.

MIRANDA, Renato. Motivação no trekking: um caminhar nas montanhas. UGF, 2000.

RIBEIRO, Ivana de C. Ecologia de corpo e alma e transdisciplinariedade em educação ambiental. UNESP/Rio Claro, 1998.

ALMEIDA, Ana C. P. C. de. O futuro das atividades fisicas de lazer e recreação ligadas à natureza e à educação ambiental. UFSC, 2000.

CHRISTOFOLETTI, João F. O corpo urbano nas trilhas da natureza. Unicamp, 1999.

ZIMMERMANN, Ana C. Atividades fisicas de aventura na natureza: elementos teórico-práticos dessas atividades na ilha de Santa Catarina. UFSC, 2001.

ANDRADE, Marcelo de M. Lazer esportivo: fundamento da ecologia humana urbana. USP, 1992.

VILLAVERDE, Sandoval. Da natureza do espaço ao espaço da natureza: reflexões sobre a relação corpo-natureza em parques públicos urbanos. Unicamp, 1999.

MARINHO, Alcyane. Da busca pela natureza aos ambientes artificiais: reflexões sobre a escalada esportiva. Unicamp, 2000.

ABDALAD, Luciana S. A participação feminina nos esportes de aventura e risco: um vôo no universo do desafio e da incerteza. Universidade Gama Filho, 2001. 
LADISLAU, Carlos R. Lazer e meio ambiente: percorrendo as trilhas de Ouro Preto (um estudo a partir das trilhas do Pico do Itacolomi). Unicamp, 2001.

PIMENTEL, Giuliano G. de A. Ações motrizes e representações sociais no jogo do laço no vale do Itabapoana. Unicamp, 2001.

FERREIRA, Luiz F. S. Corridas de aventura: construindo novos significados sobre corporeidade, esportes e natureza. Unicamp, 2003.

RECHIA, Simone. Parques públicos de Curitiba. Unicamp, 2003.

VILLAVERDE, Sandoval. Modernidade, formas de subjetivação e amizade: potencialidades das experiências de lazer e aventura na natureza. Unicamp, 2003.

UVINHA, Ricardo R. Turismo de aventura: uma análise do desenvolvimento desse segmento na Vila de Paranapiacaba. USP, 2003.

COIMBRA, Daniele A. O significado emocional das atividades físicas de aventura na natureza na determinação do estilo de vida contemporâneo. Unimep, 2004.

PASSOS, Kátia C. M. Caminhando nas trilhas do reencantamento pela natureza - uma ecologia do corpo sagrado e errante. Universidade Gama Filho, 2004.

SILVA, Renata L. As emoções das atividades físicas de aventura na natureza e a ressignificação do papel feminino. Unesp/Rio Claro, 2004.

SOUSA, Fabiana R. O imaginário no rafting: uma busca pelos sentidos da aventura, do risco e da vertigem. Universidade Gama Filho, 2004.

SOUZA, Manoela A. Aventura e risco no pára-quedismo: um estudo do imaginário social. 2004. Universidade Gama Filho, 2004.

TAHARA, Alexander K. Aderência às atividades físicas de aventura na natureza, no âmbito do lazer. Unesp/Rio Claro, 2004.

TEREZANI, Denis R. Popularização da canoagem como esporte e lazer - o caso de Piracicaba. Unimep, 2004.

SILVA, Andréia. A natureza visitada: um estudo de caso na cidade de Santa Teresa-ES. Unicamp, 2005. 
Alguns trabalhos, em nossa visão, merecem destaque para este texto:

- VILLAVERDE, em sua dissertação e tese, respectivamente, aborda a relação Lazer - Educação, mediada pelo meio ambiente, em espaços naturais "urbanos" - os parques, e em espaços na natureza propícios à escalada.

- Os estudos de MARINHO, LADISLAU, FERREIRA, PASSOS, R. SILVA, SOUSA e A. SILVA, acabam por discutir uma forma de Educação que acontece explícita ou implicitamente nas PA's.

- E a tese de RECHIA, a qual aponta uma forma de educar a relação ser humano - natureza através do Lazer em parques e praças com áreas verdes nos centros urbanos.

\section{PERIÓDICOS, REVISTAS E LIVROS}

$\mathrm{Na}$ Motrivivência, podemos encontrar textos sobre a temática em artigos e na seção "Porta Aberta" de diversos números. Destacamos o número 22/2004, com o tema "Educação Física, esportes e meio ambiente", com importantes textos para reflexão sobre Lazer e Educação, mediados pela relação ser humano - meio ambiente.

Na Revista Brasileira de Ciências do Esporte (RBCE), o volume 12 (1, 2 e 3), de 1992, teve o "Lazer" como tema central e alguns textos discutem a sintonia Lazer e Educação, mas sem relação com o meio ambiente. Já o volume 18 (2), de 1997, apresenta texto de Heloísa Bruhns e outro de Humberto Inácio, os quais abordam possibilidades de Educação pelo Lazer através das PA's. Mais recentemente, Fernando Mascarenhas (v. 24, n. 3), mesmo sem se referir especificamente às relações entre lazer/educação/meio ambiente, aponta caminhos significativos para debater esta temática pela via da Cultura.

A Licere (CELAR/UFMG) tem o objetivo de "difundir o conhecimento construído na área do Lazer e está aberta a profissionais, pesquisadores, grupos de trabalho/pesquisa e estudantes de diferentes formações, preocupados com o avançar da discussão sobre o Lazer em nosso contexto". ${ }^{4}$ Pode-se encontrar vários textos, ao longo de suas edições que debatem a relação aqui apresentada.

Por sua vez, na Conexões (Faculdade de Educação Física/Unicamp), destacamos o n. 3/1999, que apresenta seis provocantes textos de pesquisadores do GLEC sobre "Lazer \& Meio Ambiente". Enten- 
demos que esta edição da Conexões foi significativa para o boom da temática no seio da Educação Física.

Nos últimos anos, vários livros têm sido colocados à disposição da área:

- Viagens à natureza: turismo, cultura e ambiente, Célia Serrano e H. Bruhns (Org.).

- Olhares contemporâneos sobre o turismo, C. Serrano, H. Bruhns e Maria Tereza Luchiari (Orgs.).

- Lazer e desenvolvimento regional, Ademir Muller e Lamartine P. da Costa (Orgs.).

- Lazer e estilo de vida, Míria S. Burgos e Leila M. S. M. Pinto (Org.).

- Turismo, lazer e natureza, A. Marinho e H. Bruhns (Org.).

- Esportes de aventura e risco na montanha: um mergulho no imaginário. Vera L. de M. Costa.

- O imaginário no Rafting: uma busca pelos sentidos da aventura, do risco e da vertigem. Fabiana R. de Sousa.

- Turismo de aventura: reflexões e tendências. R. UVINHA (org.).

- E os três volumes da Coletânea Práticas corporais, ${ }^{5}$ de Ana Márcia Silva e Iara R. Damiani (Orgs.), entre muitos outros.

\section{GRUPOS DE ESTUDOS}

- Grupo de Estudos Lazer e Cultura - GLEC (Unicamp)

Coordenadora: Heloísa Turini Bruhns

Pesquisadores atuais ou que já passaram pelo grupo: Simone Rechia, Sandoval Villaverde, Alcyane Marinho, Giuliano Pimentel, Luiz F. Ferreira, Andréia Silva, Marcos Bettine, Wilson Lino de Souza, Ricardo Uvinha, Carlos R. Ladislau, Giuliano Pimentel, Silvia Amaral, Cheng Hsin Chao, Cristiane Kerr de Melo, entre outros.

O enfoque teórico do grupo volta-se especialmente às questões socioculturais, relacionando lazer, corpo e motricidade. No âmbito destas questões, é possível observar atualmente nos trabalhos do grupo uma aproximação aos temas que englobam a questão do meio ambiente, seja abordando práticas corporais de lazer e aventura em ambientes naturais, ecoturismo, educação ambiental, entre outros (BRUHNS et al., 2000, p. 98). 
Principais contribuições à produção do conhecimento na temática específica:

Revista Conexões, n. 3, 1999, temática "Lazer \& Meio Ambiente". Artigos de seis pesquisadores do GLEC.

XI CONBRACE, 1999, quatro trabalhos de pesquisadores do grupo.

XII CONBRACE, 2001, cinco trabalhos de pesquisadores do grupo.

Apresentações constantes de suas pesquisas nos ENAREL's.

- Laboratório de Estudos do Lazer - LEL (UNESP - Rio Claro)

Coordenadora: Gisele Maria Schwartz.

Pesquisadores atuais ou que já passaram pelo grupo: Alcyane Marinho, Fernanda Machado, Alexander Tahara, Viviane Dias, Juliana Ruiz, Mara Cristan, Marília Freire, Paul T. Jonson, Tereza França, Rita de Cássia Fenalti, Josset C. de Gáspari, Renata L. Silva, Gustav M. Luba, Tatiana P. das Neves, Rosa M. Simões, José A. Santos Filho, Pedro F. Felício.

Destacamos os principais temas abordados pelo LEL:

Psicologia do lazer [...]

Educação para o lazer [...]

Lazer e Meio Ambiente

- sensibilização e contemplação

- ecolazer e ecoturismo

- esportes na natureza

- formação de monitores de ecolazer

- lazer e educação ambiental

Lazer e Atividades Recreativas Especiais

Lazer e Qualidade de Vida (SCHWARTZ; MARINHO, 2001, p.132-133).

Principais contribuições à produção do conhecimento na temática específica:

Curso de Extensão Universitária "Vivências em atividades de aventura".

Mesa-redonda "Lazer, Natureza e Desenvolvimento Humano", no II Congresso Internacional de Educação Física e Motricidade Humana e VIII Simpósio Paulista de Educação Física (Rio Claro, 2001). 
Encontro "Grupos de Estudos, Pesquisas e Intervenção na área de Lazer", no evento supracitado.

Apresentações constantes de suas pesquisas nos ENAREL's e CONBRACE's.

- Núcleo Interdisciplinar de Estudos do Lazer - NIEL (UFPE)

Coordenadora: Tereza França.

Pesquisadores atuais ou que já passaram pelo grupo: Francisca Bion, Edilson F. de Souza, Kátia B. Cavalcanti, Pereira de Melo, Bartolomeu F. de Medeiros, Edmilson Pires, Elizabeth Jatobá, Tereza L. de França, Sávio Assis, Rosa M. A. Simões, Ana L. F. dos Santos, Alcyane Marinho, Warlindo Carneiro, Daise L. de A. França, Fernando Melo, Laurecy D. Santos, Maria C. de Albuquerque, Rosângela C. B. Lindoso, Gisele Schwartz.

Linhas de Pesquisa: Formação Profissional, Prática Pedagógica, Políticas Públicas e Corporeidade e Lazer; nestas linhas integram unidades de pesquisas, a saber:

- Escola e prática pedagógica no âmbito do Lazer/recreação.

- Políticas públicas para o Lazer/recreação.

- O trabalhador: seu Lazer e seu trabalho.

- Lazer e esporte e aventura.

- Práticas lúdicas: dimensões da corporeidade do idoso para melhoria da qualidade de vida. (Projeto ensino-pesquisa-extensão NIEL, p.14, mimeo).

Principais contribuições à produção do conhecimento na temática específica:

Apresentações constantes de suas pesquisas nos ENAREL's e CONBRACE's.

- LEPEL- Linha de Pesquisa Educação Física, Esporte e Lazer (UFBA). A LEPEL não é um grupo como os outros anteriormente apresentados, mas sim uma linha inserida no Programa de Pós-Graduação em Educação da UFBA. Sua atuação e trabalhos desenvolvidos justificam sua apresentação neste esboço de estado da arte.

Coordenadora: Celi Taffarel 
Pesquisadores atuais ou que já passaram pelo grupo: Admilson Santos, Amélia V. Conrado, Augusto C. R. Leiro, Carlos R. Colavolpe, Francisco J. Pitanga, Fátima M. Garcia, Geraldo Barroso Filho, Hélio José de Campos, José N. Santos, Lauro P. Xavier Neto, Micheli Escobar, Márcia F. Chaves, Pedro Abib, Romilson A. dos Santos. Soraia Domingues, Cláudio Lira, entre outros.

\section{Linhas de Pesquisa:}

Esporte adaptado, Atividade Física e Saúde, Capoeira, Corpo, Memória e Cultura, Esporte, Ginástica, Lazer, Educação Física \& Esporte e Lazer.

Objetivos: Repercussões na formação acadêmica, inicial, continuada e em serviço, na produção teórica, na constituição de redes de intercâmbio acadêmico, na editoração, publicação e nas políticas públicas na área de educação, educação física \& esporte e lazer.

Intercâmbio institucional com IFES da região - UFPE, UFS, UFAL e com as seis Instituições de Ensino Superior no Estado da Bahia que mantêm Cursos de Educação Física.

- BACOR- Base de Pesquisa Corporeidade e Educação (UFRN)

Seus líderes são Edmilson Ferreira Pires e Edson Ferreira Claro.

Os pesquisadores do grupo são: Edmilson P. Albuquerque, Elizabeth J. Tinoco, João E. Gomes, Pierre N. G. da Silva, Teodora de A. Alves, Tereza França, Vera L. de M. da Costa, Vera U. Lins, Kátia B. Cavalcanti, Ágrio de O. Chacon Filho e Keila B. Cavalcanti.

Linhas de pesquisa: Corporeidade, Arte e Expressividade $\mathrm{Hu}$ mana, Corporeidade, Cotidiano e Qualidade de Vida, Corporeidade, Escola e Instituições Educativas, Corporeidade, Lazer, Ecodesenvolvimento e Políticas Públicas, Corporeidade, Ludicidade e Imaginário, Corporeidade, Trabalho e Profissionalização e Fundamentos Teóricos e Metodológicos da Corporeidade.

O grupo apresenta o conceito de Ecolazer, reflexo de suas pesquisas envolvendo a temática ambiental e de um referencial teórico pou- 
co conhecido na área da Educação Física: o Ecodesenvolvimento e as obras de Henrique Leff.

- GPL- Grupo de Pesquisas em Lazer (UNIMEP-Piracicaba)

São líderes os professores Nelson C. Marcellino e Hélder F. Isayama. É importante destacar que Marcellino constitui-se uma das principais referências para os estudiosos do lazer no Brasil, desde a década de 1980. Uma de suas primeiras obras, "Lazer e Educação", repercutiu de forma inquestionável nas pesquisas, projetos e na própria formação profissional em Educação Física ao longo das duas últimas décadas. Seguindo a tendência de estudos envolvendo a relação ser humano - natureza através de práticas corporais de aventura, recentemente vem orientando trabalhos nesta direção.

Ainda fazem parte: Felipe Barbosa, Mirleide Chaar Bahia, Agostinho M. Bonfim, Marcolino Malosso Filho, Denis R. Terezani, Mar$\cos$ R. Minutti, Edmur Stoppa, Maria Z. Koritiak, Mônica Delgado, Ignácio C. Izquierdo, Oldrey P. Gabriel, José L. de Paiva, Silvio R. da Silva, Jossett Campagna, Tânia M. Sampaio e Liana A. Romera, entre outros.

A linha de pesquisa é "Corporeidade e Lazer".

Principais contribuições à produção do conhecimento na temática específica:

Nas duas últimas edições dos ENAREL's e dos CONBRACE's este grupo tem apresentado vários trabalhos referentes à temática, com uma forte ampliação na edição 2005 do Enarel. Lazer

- LIRES-LEL - Imaginário Social em Ludicidade, Esportes e

\section{Líder: Vera Lúcia de Menezes Costa}

Este grupo não era muito conhecido dos pesquisadores mais ligados à temática do lazer que sistematicamente participam dos ENAREL's e CONBRACE's. A partir do CONBRACE/2003 alguns trabalhos tornaram o grupo presente e suas contribuições foram/são significativas para a relação lazer, meio ambiente e educação. Em especial, destaca- 
mos a dissertação e o livro de Fabiana R. Sousa e o livro da líder do grupo, ambos já citados.

Participam deste grupo: Nilda Teves Ferreira, Maria Auxiliadora Cunha, Maria Regina de Menezes Costa, entre outros.

Suas linhas de pesquisa são: Análise de Componentes da Ludicidade nos Esportes de Aventura e Risco, Análise dos Aspectos Simbólicos dos Jogos Populares e Aventura, Risco e Vertigem em Esportes de Portadores de Deficiências.

Ainda que haja outros grupos e núcleos de pesquisa envolvidos com a temática aqui apresentada, entendemos que estes anteriormente listados são os mais destacados em pesquisas e projetos vinculando lazer - meio ambiente e educação.

\section{CONCLUINDO}

Sobre "Lazer e Educação", suas relações, possibilidades de interação e potencial pedagógico implícito no lazer, já se vem "conversando" há algum tempo. Sem dúvida, desde a década de 1980 até agora muito se avançou: outros pesquisadores surgiram, novas perspectivas foram anunciadas, grupos e núcleos de pesquisas se constituíram.

Por outro lado, a inserção da questão ambiental é mais recente. Assim como outras áreas do conhecimento, a Educação Física, nas suas diversas dimensões e possibilidades, tem se prontificado ao enfrentamento de questões ambientalmente relevantes para a humanidade e para o planeta. A parceria com a Sociologia, a Antropologia, o Turismo, a Geografia e outras áreas, só tem feito enriquecer e avançar a produção da temática.

Para nós, está claro que é na dimensão do Lazer que este debate se pronuncia. Algumas novidades, como as contribuições de Maffesoli e Leff, ou a apresentação dos conceitos de Ecodesenvolvimento e Ecolazer, nos instigam na busca do que pode representar o debate que envolve Lazer e Educação, em termos de uma outra formação humana, na direção de transformações sociais, mediados por práticas não formais, não tradicionais, não esportivizadas (pelo menos ainda). Mas ainda há o que fazer. No Seminário "Educação Física e a Questão Ambiental” no 
CONBRACE/2003, apuramos outras perspectivas que podem/devem ser objeto de curiosidade e investigação, algumas delas:

Identificar as matrizes epistemológicas dos estudos desenvolvidos nesta temática; a inserção desta temática nas grades curriculares dos cursos de formação; o cruzamento entre teorias e autores; as representações da relação ser humano - natureza nos meios de comunicação; a inserção das PA's no âmbito da Educação Física Escolar, entre outras.

Buscamos neste paper apresentar algumas indicações, caminhos e alertas que impulsionem pesquisadores de todos os níveis e áreas interessados no Lazer, na Educação e na urgente questão ambiental a trilhar estes caminhos. "Tentamos trazer elementos que possam contribuir para a construção de outra concepção paras as PA's, para a educação, e quiçá para nossa percepção da Natureza e do desenvolvimento de nossa civilização" (INÁCIO et al., 2005, p. 85).

\title{
Leisure, education and the environment: an adventure still under development
}

\begin{abstract}
This text intends to give a few contributions to the research theme of Leisure and Education with special attention given to the relation between leisure and the environment, mainly through Outdoor Body Practices, by presenting researcher's productions publicized in the Brazilian Congress of Sports Sciences and the National Recreation and Leisure Meeting. This article also presents the contributions from theses, dissertations, periodicals and books in the field of Physical Education or in closely related fields. Lastly, it lists research groups that have been conducting research and projects related to leisure, education, and the environment.
\end{abstract}

KEYWORDS: physical education - leisure - education - the environment - outdoor adventure practices.

Ocio, educación y medio ambiente: una aventura en construcción

\section{RESUMEN}

Este texto busca demostrar algunas contribuciones de la temática Ocio y Educación, con atención a la relación entre ocio y medio ambiente, principalmente a través de las Prácticas Corporales en la Naturaleza, presentando las producciones de investigadores divulgadas en el Congreso Brasileño de Ciencias del Deporte y en el Encuentro Nacional de Recreación y Ocio. El artículo también presenta las contribuciones de tesis, disertaciones, periódicos y libros del área de la Educación Física o próximos de ella. Finalmente, lista grupos de investigación que viene desarrollando investigaciones y proyectos relacionando ocio, educación y medio ambiente. 
PALABRAS-CLAVE: educación física - ocio - educación - medio ambiente - prácticas de aventura en la naturaleza

\section{NOTAS}

1 As PA's têm-se constituído tanto em práticas de lazer quanto em atividades profissionais, notadamente quando esportivizadas. Recebem denominações diversas: esportes ecológicos, esportes radicais, esportes de aventura, ecoturismo, atividade física de aventura na natureza, polissemia essa que, por si só, já demonstra a grande complexidade envolvida na temática.

2 Este texto tem origem no Seminário "Educação Física e a Questão Ambiental", ministrado no XIII CONBRACE/2003. As contribuições partilhadas naquele seminário, além das sugestões para tornar público o conteúdo do mesmo, somadas à oportunidade da temática deste número da Revista Pensar a Prática, é que nos impulsionaram a fazê-lo.

3 Em 2005, o ENAREL foi realizado no mês de novembro, portanto, fora de um prazo possível para análise neste paper.

4 (http://www.lazer.eefd.ufrj.br/licere/home.htm, acesso em 27/09/2005)

5 Volume 1 - Práticas corporais: gênese de um movimento investigativo em Educação Física. Volume 2 - Práticas corporais: Trilhando e Compar (trilhando) as ações em Educação Física. Volume 3 - Práticas corporais: experiências em Educação Física para outra formação humana.

\section{REFERÊNCIAS}

BRUHNS, H. T.; MARINHO, A. (Org.). Turismo, lazer e natureza. Barueri: Manole, 2003.

BRUHNS, H. T.; MARINHO, A.; RECHIA, S.; VILLAVERDE, S. Grupo de Estudos Lazer e Cultura - GLEC. Motrivivência, Florianópolis, ano 11, n. 15, p. 97-101, ago. 2000.

BURGOS, M. S.; PINTO, L. M. S. M. (Org.). Lazer e estilo de vida. Santa Cruz do Sul: Edunisc, 2002.

CONBRACE. Congresso Brasileiro de Ciências do Esporte, 12. Anais. Caxambu: Secretaria Estadual/CBCE de São Paulo, 2001. CD ROM. 
CONBRACE. Congresso Brasileiro de Ciências do Esporte, 13., Anais. Caxambu: CBCE, 2003. CD ROM.

. Congresso Brasileiro de Ciências do Esporte, 14., Anais. Porto Alegre: CBCE, 2005. CD ROM.

CONEXÕES. Educação, esporte, lazer. Campinas: Faculdade de Educação Física-UNICAMP, n.3, dez/1999.

ENAREL. Encontro Nacional de Recreação e Lazer, 11., 1999. Lazer, meio ambiente e participação humana. Cascavel: Assoeste, 1999.

. Encontro Nacional de Recreação e Lazer, 12., 2000. Formação profissional no lazer: perspectivas e tendências. Balneário Camboriú: Roca, 2000.

. Encontro Nacional de Recreação e Lazer, 13., 2001. Lazer, transdisciplinaridade e educação. Natal: Centro de Educação Tecnológica do Rio Grande do Norte, 2001. CD ROM.

. Encontro Nacional de Recreação e Lazer, 14., 2002. Lazer: desenvolvimento regional e estilo de vida. Santa Cruz do Sul: UNISC, 2002. CD ROM.

. Encontro Nacional de Recreação e Lazer, 15., 2003. Lazer e Trabalho: novos significados na sociedade contemporânea. Santo André: Prefeitura Municipal de Santo André, SESC, 2003. CD ROM.

. Encontro Nacional de Recreação e Lazer, 16., Lazer como cultura: o desafio da inclusão. 2002. Salvador: SESI, UFBA, 2004. CD ROM.

. Encontro Nacional de Recreação e Lazer, 17., Lazer e Ética. Campo Grande: UCDB, 2005. CD ROM.

INÁCIO, H. L. D.; SALLES e SILVA, A. P.; PERETI, É. S.; LIESENFELD, P. Experiências em educação física para outra formação humana. In: SILVA, A. M.; DAMIANI, I. R. (Orgs.). Práticas corporais. Florianópolis: Nauemblu Ciência e Arte, 2005. p. 69-87.

LICERE. Revista do Centro de Estudos de Lazer e Recreação/EEF/ UFMG. Belo Horizonte: CELAR. v. 3, n. 1, 2000.

. Revista do Centro de Estudos de Lazer e Recreação/EEF/UFMG. Belo Horizonte: CELAR. v. 4, n. 1, 2001. 
MARX, K. O trabalho alienado. Manuscritos econômico-filosóficos. Lisboa: Edições 70, 1989.

MASCARENHAS, F. O pedaço sitiado: cidade, cultura e lazer em tempos de globalização. Revista Brasileira de Ciências do Esporte, Campinas, v. 24, n. 3, p.121-143, 2003.

MOTRIVIVÊNCIA. Educação Física, Corpo e Sociedade (I). Ano 11, n. 15. Florianópolis: UFSC. ago. 2000.

. Educação Física, Corpo e Sociedade (III). Ano 12, n. 16. Florianópolis: UFSC. mar. 2001.

REVISTA Brasileira de Ciências do Esporte. ano 21, n. 1, caderno 2. Anais do Congresso Brasileiro de Ciências do Esporte, 11. Ijuí: Sedigraf, set. 1999.

. Lazer. v.18, n. 2. Ijuí: Unijuí, 1997.

SOUSA, F. R. O imaginário no rafting: uma busca pelos sentidos da aventura, do risco e da vertigem. São Paulo: Zouk, 2004.

SCHWARTZ, G. M.; De GÁSPARI, J. C. GTT Recreação e Lazer: tendências temáticas. In: CONGRESSO BRASILEIRO DE CIÊNCIAS DO ESPORTE, 13., 2003, Caxambu. Anais... Caxambu: CBCE, 2003. CD-ROM.

SCHWARTZ, G. M.; MARINHO, A. Laboratório de Estudos do Lazer. Motrivivência, Florianópolis, ano 12, n.16, p. 131-135, mar. 2001.

Recebido: 10 de outubro de 2005 Aprovado: 10 de dezembro de 2005

Endereço para correspondência: Rua João do Valle Pereira, 183 - João Paulo Florianópolis - Santa Catarina CEP 88030-370 E-mail: humberto@ufpr.br 
